\title{
QUINZE ANOS DO PROGRAMA DE P\&D DA ANEEL: UM ESTUDO BIBLIOMÉTRICO
}

\author{
FIFTEEN YEARS OF R\&D ANEEL PROGRAM: A BIBLIOMETRIC STUDY
}

Recebido em 11.04.2016. Aprovado em 20.10.2016

Avaliado pelo sistema double blind review

DOI: http://dx.doi.org/10.12712/rpca.v10i4.753

\section{Camila Franco}

camila.franco@coppead.ufrj.br

Instituto COPPEAD de Administração - Universidade Federal do Rio de Janeiro (UFRJ), Rio de Janeiro/RJ, BRASIL

\section{Resumo}

O objetivo deste artigo é realizar uma busca na literatura para encontrar os trabalhos publicados sobre os projetos de P\&D desenvolvidos pelas empresas do setor elétrico. Para isso foi utilizada a técnica da bibliometria com auxílio do software R. Os principais resultados obtidos nesta busca informam que a maioria dos trabalhos encontrados se caracteriza como dissertações de mestrado e artigos apresentados em congressos, o que retrata a pouca difusão deste tópico entre os periódicos científicos. Outros achados importantes referemse à concentração geográfica de tais trabalhos; aos impactos que eles apresentam, medido pelo número de citações; e aos temas de projetos analisados, em sua maioria referente à Avaliação e Gestão dos Projetos. Estes resultados proporcionam um panorama geral sobre o campo de pesquisa estudado e fornecem insights para futuros trabalhos e contribuições efetivas para a política inovativa no setor.

Palavras-chave: Inovação. P\&D. Setor Elétrico. Projetos

\section{Abstract}

The objective of this article is do a literature search to find works published About the R\&D projects developed by Electric Sector Companies. For this was used a bibliometrics technique with software assistance R paragraph. The main results obtained this search inform that most found work characterized as dissertations and papers presented at congresses, what mean a little diffusion this topic between the scientific journals. Other important findings refer to the geographical concentration of such works; to impacts que they have, measured by number of citations; and to the project themes analyzed, mostly concerning the Assessment and Project Management. These results provide a general panorama about the field studied and provide insights for future work and effective contributions to Innovative Policy not Sector.

Keywords: Innovation. R\&D. Power Sector. Projects 


\section{Introdução}

O papel da inovação no contexto econômico é cada vez mais fundamental para as empresas em diversos setores, já que se mostra como uma maneira de diferenciarem-se e tornarem-se competitivas no mercado atual. De acordo com Atalay et al (2013) a inovação é considerada como uma das mais importantes fontes de vantagem competitiva sustentável, pois contribui para melhorias de produtos e processos, permite avanços contínuos que ajudam as empresas em questões ligadas a sobrevivência, crescimento, eficiência e rentabilidade.

Para serem competitivas e alcançar destaque é preciso que haja uma preocupação com as questões que podem levar a vantagens significativas. $\mathrm{Na}$ maioria das vezes, essas questões são associadas ao processo de inovação, fator que mantém ou não determinadas empresas no cenário competitivo global.

Tendo em vista a importância deste fator para a sobrevivência das organizações, o governo federal brasileiro promulgou em 24 de julho de 2000 a Lei 9.991 que obriga as empresas de energia elétrica a investir uma porcentagem de sua Receita Operacional Líquida em Projetos de Pesquisa e Desenvolvimento (P\&D), com isso surge o Programa de P\&D da ANEEL (Agência Reguladora do Setor) a fim de estimular o setor elétrico brasileiro em atividades voltadas à inovação e assim torná-lo competitivo (BRASIL, 2000).

Em 15 anos de promulgação da Lei diversos trabalhos acadêmicos já foram desenvolvidos com propostas diferentes, analisando o Programa de P\&D. O objetivo desta pesquisa é buscar os trabalhos publicados sobre estes projetos desde o ano da promulgação da lei até os dias atuais na tentativa de entender as nuances relacionadas a tais pesquisas.

Para atingir este objetivo será realizada uma pesquisa bibliométrica em algumas das principais bases de dados acadêmicas e analisados os indicadores sugeridos na literatura a fim de garantir a robustez da técnica. Para analisar os dados será utilizado o software estatístico $\mathrm{R}$ com alguns pacotes internos que fornecem as ferramentas necessárias para tal.

A principal contribuição deste trabalho é proporcionar uma visão geral do que já foi pesquisado sobre os projetos de $\mathrm{P} \& \mathrm{D}$ do setor elétrico brasileiro e assim fornecer insigths para futuras abordagens e novos descobrimentos sobre este Programa.
A estrutura deste artigo compreende, após esta introdução, uma discussão sobre Inovação e o setor a ser estudado; em seguida é apresentada a metodologia utilizada, seguida pela apresentação e discussão dos resultados, e encerrando-se com as considerações finais e sugestões para pesquisas futuras.

\section{Referencial Teórico}

\section{Inovação}

A inovação é um tema que se discute há bastante tempo no meio acadêmico. Uma variável que cresce em importância no ambiente organizacional pelo seu caráter de constante mudança. Segundo Pavitt (1990), algumas das principais características que definem esta variável são: a incerteza, o risco, e a não linearidade. Contudo, embora seja um fator que apresente características de instabilidade, a inovação representa uma das principais fontes de vantagem competitiva para as empresas no cenário econômico atual.

Schumpeter (1942), um dos pioneiros a discutir sobre inovação na literatura, já afirmava que esta representa um fenômeno fundamental para o desenvolvimento econômico e que a empresa é um agente importante de inovações, cujas relações internas e externas deliberam o seu processo evolucionário.

$\mathrm{O}$ autor argumenta que, para se entender o processo de inovação é preciso que se faça a distinção entre os termos invenção e inovação, muitas vezes confundidos e tratados como sinônimos. Schumpeter (1942) diz que a invenção é a criação de um novo artefato que pode ou não ter relevância econômica. A invenção só se torna uma inovação se ela for transformada em uma mercadoria ou em uma nova forma de produzir mercadoria, e que seja explorada economicamente. Por outro lado, a inovação refere-se a novas combinações de recursos já existentes para produzir novas mercadorias, ou para produzir mercadorias antigas de uma forma mais eficiente, ou ainda mesmo para acessar novos mercados.

De acordo com Dosi (1988) inovar significa um processo caracterizado pela busca, descoberta, experimentação, imitação e adoção de novos processos de produção e novas configurações organizacionais. $\mathrm{E}$ este processo envolve uma intensa colaboração e interação entre os paricipantes (Pavitt, 1990). Vonortas e Zirulia (2015), argumentam que, pelo fato das empresas não possuírem todas as informações e 
conhecimentos necessários para inovar, elas buscam na formação de parcerias uma forma de suprir esta carência e, assim, encontrar as capacidades complementares para auxiliar nos processos de desenvolvimento de novos produtos e/ou processos, no monitoramento de oportunidades de mercado e na sustentação de vantagem competitiva.

O Relatório sobre os Índices de Inovação Mundial, organizados pelo instituto INSEAD, da Cornell University considera o fator inovação "como um motor de crescimento econômico e de bemestar", mostrando-se "importante para a condução econômica e o progresso da competitividade tanto para economias desenvolvidas como economias em desenvolvimento" (INSEAD, 2014).

Com tais definições e explicações a respeito deste fator percebe-se que o mesmo tem sido essencial para a competitividade das empresas no cenário global atual. Contudo, a literatura propõe que para que as empresas implementem inovações e desenvolvam-nas dentro do ambiente organizacional é necessário conhecer a fundo este ativo intangível. De acordo com o Manual de Oslo (OECD, 2005), as inovações são classificadas de acordo com o seu tipo, configurando-se em inovações de produto, processo, organizacional, ou marketing. Por outro lado, Freeman (1988) considerou que a inovação, além de tipos distintos, também possuem diferentes graus, podendo caracterizar-se como radicais ou incrementais. Posteriormente, Henderson e Clark (1990) sugeriram que não é possível ver a inovação dentro de um espectro dual que compreende apenas dois graus de intensidade, e sugeriu que existem ainda as Inovações Modulares e as Inovações Arquiteturais.

Seguindo aos conceitos abordados na literatura sobre inovações, as discussões prosseguem sobre a maneira pela qual as empresas podem inserir este processo em sua cultura e assim tornarem-se mais competitivas frente a seus concorrentes. Com isso, surge uma corrente literária que aborda as capacidades tecnológicas que são necessárias para que se alcancem tais inovações. O termo Capacidades Tecnológicas, discutido por Lall (1992), significa um estoque de recursos que a empresa possui capaz de gerar e gerir as inovações. A partir deste conceito diversos trabalhos surgiram com propósito de identificar como desenvolver estes recursos e assim gerar as almejadas inovações (BELL; PAVITT, 1993, 1995; FIGUEIREDO, 2001, 2003).

A literatura aborda diversos fatores que impulsionam o processo de inovação dentro das empresas e que podem ser fundamentais para o alcance de um maior desempenho. Alguns estudiosos têm relacionado o desempenho diretamente com a capacidade de absorver novos conhecimentos do ambiente (ANDERSSON et al., 2002), que por sua vez também impactam no nível de inovação desenvolvido pelas empresas. Calantone et al (2002) afirmam que o aprendizado é um antecedente importante para a 'inovatividade' da firma, e que esta, por sua vez, exerce uma influência sobre o desempenho. Outras pesquisas também examinam o fator de conhecimento/ aprendizado para analisar a relação inovação e desempenho (por exemplo, PRICE et al 2013; CONNELL et al, 2001).

Os investimentos em Pesquisa e Desenvolvimento $(\mathrm{P} \& \mathrm{D})$ se caracterizam como uma das principais formas que as empresas encontram para incentivar o processo de inovação, ao passo que, para se realizar Pesquisa e desenvolvê-la, a empresa está empregando capital intelectual e, desta forma, impulsionando o fator conhecimento para desenvolver a inovação. Além disso, os gastos em P\&D representam uma importante medida para mensurar o processo inovativo dentro das organizações.

Com o intuito de incentivar o processo de busca, descoberta e experimentações dentro do setor elétrico brasileiro, foi regulamentada a necessidade de se investir em Pesquisa e Desenvolvimento como forma de se alcançar a inovação e promover a competitividade frente aos desafios tecnológicos.

\section{Setor elétrico brasileiro}

A indústria de energia elétrica é constituída por agentes independentes que, ou produzem, ou transportam ou comercializam a energia elétrica.

O período atual que vive o setor elétrico vem de uma reestruturação ocorrida em meados dos anos $90 \mathrm{com}$ mudanças institucionais e operacionais feitas pelo Ministério de Minas e Energia. Estas mudanças se basearam no consenso político-econômico do "estado regulador", que deveria direcionar as políticas de desenvolvimento, bem como regular o setor. Assim, muitas empresas foram privatizadas e autarquias foram criadas, como por exemplo, a Agência Reguladora do Setor - ANEEL (ABRADE, 2015).

O papel da ANEEL é proporcionar condições favoráveis para que o mercado de energia elétrica se desenvolva com equilíbrio entre os agentes e em benefício da sociedade (ANEEL, 2015). 
Com intenção de incentivar a busca constante por inovações e fazer frente aos desafios tecnológicos do setor elétrico, o governo federal brasileiro promulgou em 24 de julho de 2000 a lei 9.991 a qual estabelece que as empresas de energia elétrica brasileira devem investir uma porcentagem de sua Receita Operacional Líquida em Projetos de Pesquisa e Desenvolvimento. Para ser considerado P\&D os projetos "deverão estar pautados pela busca de inovações para fazer frente aos desafios tecnológicos e de mercado das empresas de energia elétrica" (ANEEL, 2008, p.7). Desta maneira, cabe à ANEEL regulamentar o investimento no programa, acompanhar a execução dos projetos e avaliar seus resultados.

A ANEEL é responsável por estabelecer as diretrizes e orientações que regulamentam a elaboração de projetos de P\&D por meio do Manual de Programa de Pesquisa e Desenvolvimento do Setor de Energia Elétrica. As avaliações dos resultados de tais projetos são feitas em conformidade apenas com este Manual, ou seja, são considerados satisfatórios os projetos que cumprem as metas estabelecidas nas diretrizes. Contudo, os resultados são interpretados dentro de uma ótica a responder o objetivo primeiro da promulgação da Lei, que é incentivar a inovação e o desenvolvimento tecnológico do setor. É preciso que estes resultados sejam analisados dentro de um escopo institucional que possibilite visualizar o setor dentro de uma escala de inovação e assim, ratificar se, realmente, os projetos estão possibilitando que haja desenvolvimento inovativo para o país (ANEEL, 2015).

\section{Metodologia}

Para atingir o objetivo proposto neste trabalho, será utilizada a técnica da bibliometria, que visa analisar a produção científica existente em livros, artigos apresentados em eventos, artigos publicados em periódicos e outros documentos diversos (VANTI, 2002).

No trabalho de Teixeira et al (2013) é discutido sobre a técnica da bibliometria nos estudos em Administração, os autores questionaram se realmente esta técnica tem sido utilizada de forma correta com indicadores adequados e pautados na literatura a fim de que se possa de fato contribuir para o avanço do conhecimento. Como resultados eles concluíram que os trabalhos que empregam esta técnica na Administração não são realizados de maneira a enquadrar todas as informações a que a técnica se dispõe. Com isso, os autores sugerem alguns pontos que devem ser seguidos em trabalhos desta natureza para que efetivamente o emprego da bibliometria seja feito de maneira eficaz. Estes pontos são: a) analisar as leis de Lotka, Zipf e Bradfort (referente à produtividade dos autores; à frequência de ocorrência das palavras; e à produtividade das publicações, respectivamente); b) analisar a produção do conhecimento por instituição e pela origem geográfica dos autores; c) analisar categorias relacionadas ao perfil dos autores; d) analisar a distribuição do conhecimento no tempo e no espaço, identificando a evolução da produção no tempo e a distribuição geográfica; e) identificar os autores mais influentes e as influências que recebem, mediante o levantamento dos autores mais produtivos e cocitações; f) identificar abordagens epistêmica e metodológicas dominantes e nãodominantes no campo.

Para a busca por trabalhos foi realizada uma pesquisa em três bases de dados bastante conhecidas e renomadas para este tipo de trabalho (Thomson Reuters, EBSCO e Emerald). No entanto poucos trabalhos foram encontrados nelas; para suprir esta lacuna tornou-se a fazer a busca pela ferramenta acadêmica do Google (Google Acadêmico) que fornece não apenas artigos publicados em periódicos, como também publicados em anais de congresso, livros, teses e dissertações. De fato, ao se fazer a busca utilizando esta base de dados, o número de resultados obtidos foi de dez vezes maior que nas três anteriores. No Quadro 1 são mostrados os critérios de busca utilizados para encontrar os trabalhos (critérios estes utilizados apenas no título), e a quantidade de trabalhos encontrados em cada base de dados. A delimitação do tempo foi de 2000 a 2015 (desde a promulgação da Lei). 
Quadro 1. Busca de artigos pelo título nas 4 bases de dados.

\begin{tabular}{|c|c|c|c|c|c|}
\hline CRITÉRIOS DE BUSCA & $\begin{array}{l}\text { THOMSON } \\
\text { REUTERS }\end{array}$ & EBSCO & EMERALD & $\begin{array}{l}\text { GOOGLE } \\
\text { ACADÊMICO }\end{array}$ & $\underline{\text { SOMA }}$ \\
\hline "R\&D ANEEL" & & & & 1 & 1 \\
\hline "R\&D Brazilian Power Sector" & 1 & & & 2 & 3 \\
\hline \multicolumn{6}{|l|}{ "R\&D Power Sector Brazil" } \\
\hline \multicolumn{6}{|l|}{ "Research and Development ANEEL" } \\
\hline \multicolumn{6}{|l|}{$\begin{array}{l}\text { "Research and Development Brazilian Power } \\
\text { Sector" }\end{array}$} \\
\hline $\begin{array}{l}\text { "Research and Development Power Sector } \\
\text { Brazil" }\end{array}$ & 1 & & & 1 & 2 \\
\hline "P\&D ANEEL" & & & & 15 & 15 \\
\hline "P\&D Setor Elétrico Brasileiro" & 1 & 1 & & 9 & 11 \\
\hline "P\&D Setor Elétrico Brasil" & & & & 1 & 1 \\
\hline "Pesquisa e Desenvolvimento ANEEL" & & & & 3 & 3 \\
\hline $\begin{array}{l}\text { "Pesquisa e Desenvolvimento Setor Elétrico } \\
\text { Brasileiro" }\end{array}$ & & & & 9 & 9 \\
\hline \multicolumn{6}{|l|}{$\begin{array}{l}\text { "Pesquisa e Desenvolvimento Setor Elétrico } \\
\text { Brasil" }\end{array}$} \\
\hline$\underline{\text { SOMA }}$ & 3 & 1 & 0 & 41 & 45 \\
\hline
\end{tabular}

Fonte: Elaborada pelo autor

Para analisar os trabalhos encontrados, procurou-se contemplar os itens abordados por Teixeira (2013) e atender às questões para esta pesquisa, com isso a análise levou em consideração os seguintes aspectos:

1) Para verificar o que tem sido estudado sobre este tema e a maneira como se tem trabalhado, foram analisados: o objetivo do trabalho e a metodologia utilizada;

2) Para verificar o impacto destes trabalhos foram analisados: o número de citações de cada trabalho e a classificação do periódico onde foi publicado.

3) Para conhecer quais são as instituições que pesquisados sobre determinados assuntos foi analisada a rede de relações formada a partir das instituições dos autores, utilizando para isso a técnica de Análise de Redes Sociais.

Em todo o processo de análise dos trabalhos o software utilizado foi o $\mathrm{R}$, o único pacote que precisou ser instalado para a execução de alguns comandos foi o pacote 'igraph', que possibilita desenhar a rede de relacionamentos entre os artigos.

\section{Análise Dos Resultados}

\section{- Análise Quantitativa}

No Quadro 2 são mostrados todos os trabalhos encontrados, organizados por data. Percebe-se que o número total de trabalhos diverge do Quadro 1, isso se dá por que alguns trabalhos apareceram em mais de uma 
base de dados e também se repetiram em mais de um critério de busca. Outro filtro também foi necessário fazer a fim de que se pudesse começar a análise a que esta pesquisa se propõe: percebeu-se que alguns dos trabalhos encontrados não apresentavam cunho acadêmico, sendo caracterizados, por exemplo, como relatórios elaborados pela ANEEL, ou até mesmo sendo mostrado como apenas o título de uma revista, a qual não apresentava artigos acadêmicos. Com mais esta simplificação o número de trabalhos finais a serem analisados por esta pesquisa são de 31 .

Quadro 2. Total de trabalhos encontrados por data

\begin{tabular}{|c|c|c|}
\hline $\mathbf{N}^{\mathbf{o}}$ & ANO & TÍTULO \\
\hline 1 & 2000 & $\begin{array}{l}\text { Public Goods And Restructuring Of The Brazilian Power Sector: Energy Efficiency, R\&D And Low } \\
\text { Income Programs }\end{array}$ \\
\hline 2 & 2003 & $\begin{array}{l}\text { Um Estudo De Caso Para Análise Dos Projetos De P\&D Das Concessionárias Reguladas Pela } \\
\text { ANEEL }\end{array}$ \\
\hline 3 & 2003 & Pesquisa \& Desenvolvimento De Interesse Público E As Reformas No Setor Elétrico Brasileiro \\
\hline 4 & 2005 & $\begin{array}{l}\text { Power Sector Reforms In Brazil And Its Impacts On Energy Efficiency } \\
\text { And Research And Development Activities }\end{array}$ \\
\hline 5 & 2005 & Programa De P\&D Da ANEEL: Concepção, Falácias E Novos Desafios \\
\hline 6 & 2008 & $\begin{array}{l}\text { P\&D No Setor Elétrico Brasileiro: Um Estudo De Caso Na Companhia Hidro Elétrica Do São } \\
\text { Francisco. }\end{array}$ \\
\hline 7 & 2008 & $\begin{array}{l}\text { Uma Análise Fenomenológica A Respeito Da Percepção Da Inovação Nos Investimentos De Pesquisa } \\
\text { E Desenvolvimento Do Setor Elétrico Brasileiro }\end{array}$ \\
\hline 8 & 2008 & $\begin{array}{l}\text { A Contextualização Da Inovação Na Área De Pesquisa E Desenvolvimento Das Empresas Do Setor } \\
\text { Elétrico Brasileiro }\end{array}$ \\
\hline 9 & 2009 & $\begin{array}{l}\text { Transferência Do Conhecimento Tecnológico Gerado Em Projetos De P\&D No Setor Elétrico } \\
\text { Brasileiro: O Caso Eletronorte }\end{array}$ \\
\hline 10 & 2009 & Gestão De P\&D No Setor Elétrico Brasileiro: Estudo De Caso Na Duke Energy \\
\hline 11 & 2009 & $\begin{array}{l}\text { A Pesquisa E Desenvolvimento Na Estratégia Competitiva Das Concessionárias Do Setor Elétrico } \\
\text { Brasileiro. }\end{array}$ \\
\hline 12 & 2010 & Arquiteturas Organizacionais Para A Área De P\&D Em Empresas Do Setor Elétrico Brasileiro \\
\hline 13 & 2010 & Políticas Públicas De Estimulo À P\&D... \\
\hline 14 & 2010 & $\begin{array}{l}\text { Inovação, Ciência E Tecnologia No Brasil: Proposta De Avaliação De Projetos De P\&D No Setor } \\
\text { Elétrico }\end{array}$ \\
\hline 15 & 2011 & $\begin{array}{l}\text { Rede De Pesquisa Formada Pelo Programa De P\&D Regulado Pela Aneel: Abrangência E } \\
\text { Características }\end{array}$ \\
\hline 16 & 2011 & Critérios De Avaliação E Seleção De Projetos Para O Programa De P\&D Da Aneel \\
\hline 17 & 2011 & Impactos Qualitativos Do Programa De P\&D Regulado Pelaaneel \\
\hline 18 & 2011 & Impactos Econômicos E Tecnológicos Do Programa De P\&D Regulado Pela Aneel \\
\hline 19 & 2012 & $\begin{array}{l}\text { Multidisciplinary Systems Concepts Applied To R\&D Projects Promoted By Brazilian Electricity } \\
\text { Regulatory Agency (ANEEL) }\end{array}$ \\
\hline
\end{tabular}




\begin{tabular}{|c|c|c|}
\hline 20 & 2012 & $\begin{array}{l}\text { A Pesquisa E Desenvolvimento No Setor Elétrico Brasileiro: Uma Investigação Da Política } \\
\text { Tecnológica Para O Setor Com Base Na Teoria Evolucionária Da Mudança Técnica. }\end{array}$ \\
\hline 21 & 2013 & $\begin{array}{l}\text { QFD Application On Developing R\&D Project Proposal For The Brazilian Electricity Sector: A Case } \\
\text { Study - System Assets Monitoring And Control For Power Concessionaires }\end{array}$ \\
\hline 22 & 2013 & $\begin{array}{l}\text { Práticas De Gestão Da Inovação No Setor Elétrico Brasileiro: A Percepção Dos Gerentes De Projetos } \\
\text { De P\&D }\end{array}$ \\
\hline 23 & 2013 & $\begin{array}{l}\text { Quão Efetivas São Políticas Tecnológicas De Cunho Impositivas? Evidências Do Programa De P\&D } \\
\text { Capitaneadas Por Uma Empresa Do Setor Elétrico Brasileiro }\end{array}$ \\
\hline 24 & 2013 & $\begin{array}{l}\text { Investigação Apreciativa Integrada Às Práticas De Gestão Do Conhecimento Em P\&D No Setor } \\
\text { Elétrico Brasileiro: O Caso Da Companhia Hidro Elétrica Do São Francisco }\end{array}$ \\
\hline 25 & 2013 & Logística Na Apropriação De Resultados De Projetos De Pesquisa, Desenvolvimento E Inovação... \\
\hline 26 & 2013 & $\begin{array}{l}\text { Avaliação De Projetos De Pesquisa E Desenvolvimento: Influências E Desafios Do Contexto Do } \\
\text { Setor Elétrico Brasileiro }\end{array}$ \\
\hline 27 & 2013 & $\begin{array}{l}\text { Da Pesquisa E Desenvolvimento À Inovação: Um Estudo De Caso Numa Empresa Do Setor Elétrico } \\
\text { Do Nordeste Brasileiro }\end{array}$ \\
\hline 28 & 2014 & Outputs Do Programa De P\&D Da Aneel: Um Estudo Bibliométrico \\
\hline 29 & 2014 & $\begin{array}{l}\text { Processo De Desenvolvimento De Produto: Aplicação Em Um Projeto De P\&D Dentro Do } \\
\text { Programa Aneel }\end{array}$ \\
\hline 30 & 2014 & Agilidade Na Contratação De Projetos De Pesquisa... \\
\hline 31 & 2014 & Gestão De Projetos De Pesquisa E Desenvolvimento No Setor Elétrico Brasileiro \\
\hline
\end{tabular}

Fonte: Elaborada pelo autor

Antes de iniciar as análises que foram propostas para estes trabalhos, optou-se por verificar dois aspectos adicionais de importância para o entendimento das pesquisas sobre o Programa de P\&D da ANEEL: análise histórica de publicação dos trabalhos; e análise do tipo de trabalho publicado.

Em relação à análise histórica dos trabalhos publicados (Gráfico 1) nota-se que a publicação tem sido crescente, com maior frequência no ano de 2013, com sete artigos publicados, porém com duas quedas significantes de publicação (2012 e 2014). Não é possível estabelecer, com os dados possuídos, uma relação de causa e efeito a este fenômeno, apenas observar sua crescente evolução.

Gráfico 1 . Evolução Histórica dos Trabalhos pesquisados

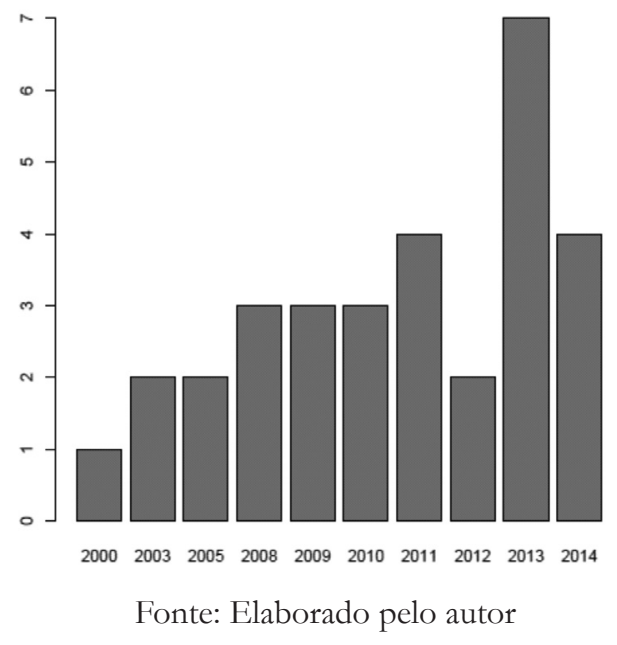


Quanto à tipologia dos trabalhos, foi realizada uma análise de frequência e verificado que 35\% das publicações foram feitas em congressos, seguida por Dissertações de Mestrado (29\%) (Gráfico 2). Com isso, verifica-se que as pesquisas não têm sido divulgadas em revistas acadêmicas, o que possibilitaria uma maior disseminação do conhecimento científico, percebe-se ainda que estes trabalhos encontrados em congressos e dissertações não foram enviados posteriormente para periódicos, confirmando a assertiva anterior.

Gráfico 2 . Distribuição dos trabalhos pelo Tipo

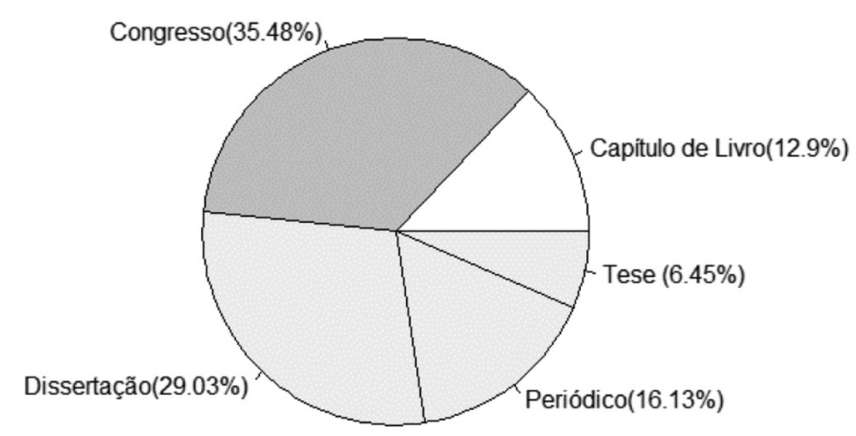

Fonte: Elaborado pelo autor

Ainda buscando entender a tipologia dos trabalhos, foi verificado, nas dissertações e teses que tratavam sobre o tema, que a maioria $(36 \%)$ advém de cursos voltados para a área de Engenharia (Industrial, de Produção e Mecânica), seguido por apenas três trabalhos $(27 \%)$ advindos do curso de Administração.

Dando início às análises propostas por este artigo, o primeiro procedimento adotado foi examinar os objetivos de cada um dos trabalhos pesquisados. Para isso, foi construída mais uma coluna no banco de dados, intitulada "Tópico Discutido" onde foi resumida a principal ideia contida no objetivo. Os resultados deste procedimento são mostrados no Gráfico 3, que demonstra que os três principais tópicos abordados nos trabalhos são: "Avaliação dos Projetos", "Gestão dos Projetos" e "Análise da Política". Em relação à Análise da Política, os trabalhos que apresentaram este tópico concentramse nos primeiros anos de publicação da lei, e foram trabalhados com intuito de investigar o quão efetiva tal política estava sendo. Quanto aos dois outros tópicos que se destacaram, observa-se que, quando se trata de Gestão dos Projetos, as pesquisas concentram-se em entender como tem funcionado o gerenciamento do
Programa de P\&D dentro das empresas; por outro lado, quando se trata de Avaliação dos Projetos, as pesquisas buscam encontrar os resultados e/ou impactos que a execução dos projetos tem gerado para as companhias. Em ambos os casos, o que se percebe é que são trabalhos específicos, focados em situações de determinadas empresas, não englobando uma visão do setor como um todo.

Gráfico 3 . Distribuição dos Temas Centrais dos Trabalhos Analisados

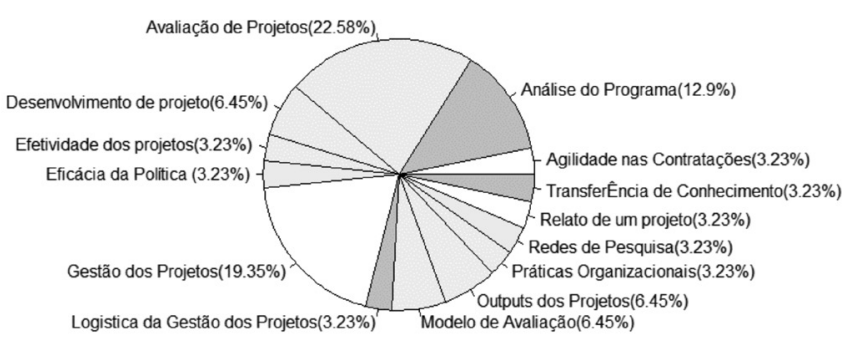

Fonte: Elaborado pelo autor

Para entender como as pesquisas têm sido realizadas para atingir os objetivos propostos, analisou-se as metodologias empregadas também através de uma análise de frequência, e verificou-se que a maioria (45\%) utilizou-se da técnica do estudo de caso. Ao concatenarmos os resultados dos tópicos discutidos com as metodologias adotadas é possível notar que os trabalhos que discutem sobre o Programa de P\&D da ANEEL, mostram-se, em sua maioria com estudos de casos únicos que buscam entender com profundidade um determinado fenômeno dentro da empresa, que se traduz em Avaliar os resultados dos Projetos ou Avaliar a Gestão dos mesmos.

Poucos trabalhos utilizaram estudos quantitativos, e com amostras grandes. Dentre os demais métodos empregados, destaca-se a incidência de um trabalho utilizando a metodologia DEA, um trabalho utilizando a técnica da bibliometria e um trabalho utilizando Análise de Variância. Outro resultado relevante quanto à metodologia é que se encontraram dois trabalhos que se apoiaram no software $\mathrm{R}$ para análise dos dados, um deles apresentou apenas estatísticas descritivas e o outro utilizou teste de correlação.

Para observar o impacto que os trabalhos pesquisados têm, a princípio pensou-se em verificar tanto o número de citações quanto o fator de impacto dos 
periódicos onde os mesmos foram publicados. Todavia, se percebeu, a partir da análise dos tipos de trabalhos que a maioria configura-se como artigos de congressos ou dissertações de mestrado. Apenas três trabalhos foram publicados em periódicos, sendo assim, a análise do fator de impacto não se enquadrou como pertinentes.

Contudo, ainda foi possível analisar o número de citações que cada trabalho recebeu. Na Figura 1 é apresentada tal relação, que destaca que um trabalho em particular atingiu mais de quarenta citações desde que foi publicado (2005); seguido deste, o próximo artigo que também apresenta um grande número de citações foi publicado 5 anos depois e recebeu quinze citações. Sobre estes dois trabalhos que se destacaram, ambos foram publicados em periódicos, sendo um deles internacional (Energy Policy) com fator de impacto de 2.57 e o outro nacional (RAC) com qualificação QUALI A2. Este achado demonstra que, embora existam poucos trabalhos nesta área publicados em revistas científicas, nota-se que quando existe algum a busca por ele retrata-se de forma satisfatória, o que leva a deduzir que o tema de pesquisa é relevante e de interesse da comunidade acadêmica, embora recente.

Ainda em relação aos dois artigos de maior impacto observou-se que ambos são do mesmo autor (Gilberto de Martino Jannuzzi) o qual trabalhou no Ministério de Minas e Energia e atualmente trabalha como professor no Departamento de Energia da Universidade de Campinas. Estes resultados fornecem uma descoberta sobre um pesquisador relevante na área e que pode auxiliar para o desenvolvimento de pesquisas futuras para aqueles que se interessem em pesquisa sobre o processo de inovação no setor elétrico brasileiro.

Para analisar a inter-relação entre as instituições que pesquisam sobre este assunto foi montada uma rede com auxílio do R, com o pacote igraph (Figura 1).
Figura 1 . Rede de Cooperação entre as Instituições

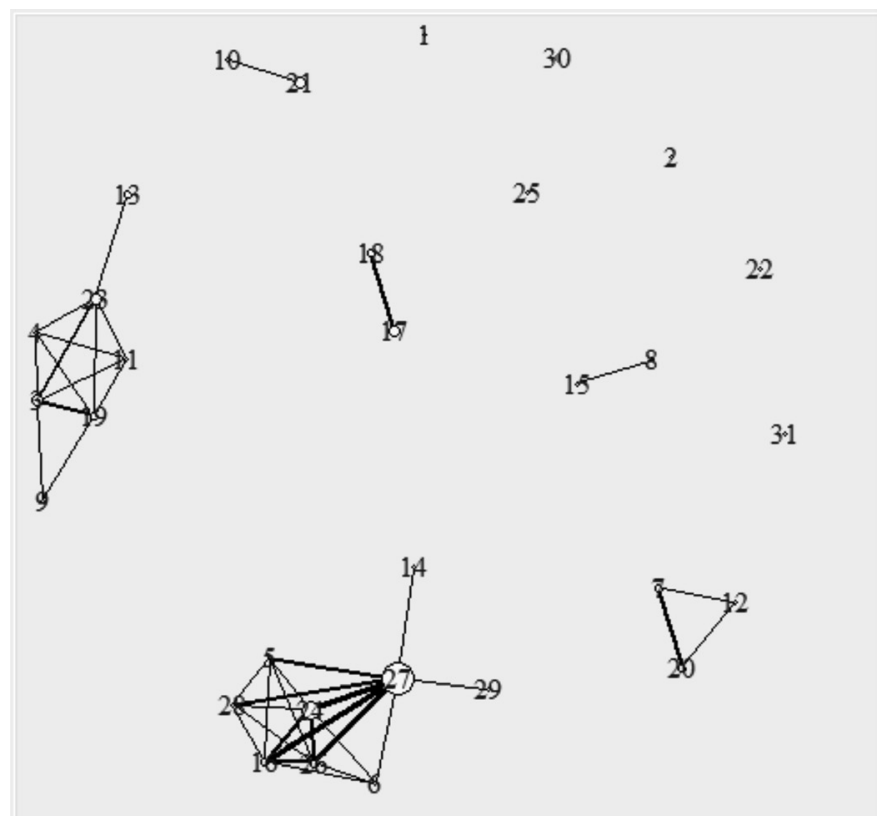

Fonte: Elaborado pelo autor

O que se percebe a partir da rede formada pelas instituições é que existe um ator que se destaca com maior número de publicações (ator 27), o qual é caracterizado pela Unicamp, que por sua vez é a instituição onde o autor Jannuzzi leciona. Outro fator importante a ser destacado a partir da análise da rede é que as instituições, em sua maioria, não fazem conexões para publicação de trabalhos. São identificados apenas dois blocos com cooperações, e mesmo nestes não se percebe que a cooperação é constante (traduzida pela grossura da linha). Todavia, foi positivo realizar tal análise e notar que há um ator principal que, além de aparecer com grande frequência de trabalhos, demonstra cooperação com outras instituições, mesmo que sejam em trabalhos pontuais.

A partir desta rede as instituições podem identificar os potenciais atores que pesquisam sobre este tema e construir cooperações para desenvolver discussões e aprimoramentos da pesquisa. 


\section{- Análise Qualitativa}

Ao decorrer desta investigação, lendo os trabalhos encontrados (além das estatísticas demonstradas até aqui), outros achados despertaram interesse a respeito do conteúdo desta discussão. Alguns trechos foram separados para suscitar determinados pontos de discussão importantes a serem avaliados neste setor:

pouca valia estará tendo a política implementada pela ANEEL se os projetos não estiverem gerando soluções aplicáveis, inovações propriamente ditas [...] As justificativas apresentadas para a não implementação dos produtos oriundos da P\&D está a incompatibilidade das tecnologias desenvolvidas com os sistemas em uso da empresa, o que demonstra falta de planejamento e de interação dos gestores de $\mathrm{P} \& \mathrm{D}$ com as demais áreas técnicas das empresas reguladas (GUEDES, 2010, pg. 100).

"Dificuldade dos gestores da empresa de deixar uma cultura escassamente orientada à inovação para adotar um novo paradigma que realmente internalize os aspectos principais de uma estratégia inovativa" (CAIAFFA, 2013).

"De acordo com a metodologia de Pavitt a inovação no setor depende de um encadeamento tecnológico entre as concessionárias, empresas formadoras de equipamento e tecnologias, universidades e ICT's" (AMARAL, 2012).

Estes depoimentos denotam que o setor de energia elétrica ainda precisa desenvolver bastante sua área de inovação, a fim de fazer valer os investimentos feitos para estimular este aspecto. Não é possível que se mantenha um pensamento como o predominante no início do Programa, de que os projetos eram realizados apenas como forma de cumprir a lei (SILVA JR; PROCOPIUK; QUANDT, 2009).

Os argumentos apresentados aqui comungam com a discussão inicial sobre o processo de Inovação. Quando Schumpeter (1942) faz a diferença entre os conceitos de invenção e inovação, deixa claro que, para se tornar inovação, é preciso que o produto/ serviço, tenha uma aplicabilidade, ultrapassando as barreiras da empresa onde o mesmo foi desenvolvido. Enquanto as concessionárias não desenvolverem inovações, o objetivo para o qual a lei foi criada não estará sendo cumprido.

Outro ponto que merece destque nesta discussão é que por apresentar características de incerteza, risco e não linearidade (Pavitt, 1990), o processo de inovação, por vezes pode não ser bem visto pelos gestores, que terão de sair de suas zonas de conforto e adotar um novo paradigma para desenvolvimento de produtos/ serviços e processos.

Além disso, para que a Inovação se faça realmente presente no setor elétrico, ou em qualquer outro setor, um ponto fundamental é que exista cooperação entre os atores imersos no ambiente. Como já afirmava Schumpeter (1942) quando dizia que a empresa é um agente de inovações cujas relações internas e externas deliberam seu processo evolucionário. Para inovar, não é possível mais se manter isolado, quer seja em seu ambiente externo, em meio a fornecedores, clientes e até mesmo competidores, quer seja em seu meio interno, conversando com os membros da organização como um todo (Pavitt, 1990; Vonortas e Zirulia, 2015).

Estimular e desenvolver o processo inovativo dentro de qualquer setor da economia significa buscar por uma competitividade de mercado que pode levar a empresa ao alcance de inúmeros benefícios em seu desempenho, como destaca Price (2013) sobre a relação positiva que existe entre a inovação e o desempenho organizacional; e Pavitt (1990) ao afirmar que a inovação representa uma fonte de vantagem competitiva para a empresa, o que garante a ela destacar-se frente aos concorrentes por meio de novos produtos e serviços.

Além disso, algumas sugestões para pesquisa futuras foram observadas nos trabalhos analisados (Quadro 3 ), este ponto pode ser fundamental para auxiliar no desenvolvimento das pesquisas e expansão das discussões sobre este tema. 
Quadro 3 . Sugestões para Pesquisas Futuras advindas dos trabalhos encontrados

\begin{tabular}{|l|l|}
\hline ARTIGO & SUGESTÕES FUTURAS \\
\hline 20 pg. 110 & $\begin{array}{l}\text { "A base do conhecimento na qual se apoia o } \\
\text { setor bem como sua trajetória tecnológica } \\
\text { precisa ser avaliado para possibilitar } \\
\text { a compreensão das competências } \\
\text { estabelecidas e sua dinâmica evolutiva". }\end{array}$ \\
\hline 22, pg. 15 & $\begin{array}{l}\text { "Destaca-se que as empresas do setor } \\
\text { elétrico devem implementar iniciativas } \\
\text { urgentes para mensurar os resultados do } \\
\text { esforço de P\&D e inovação". }\end{array}$ \\
\hline 26, pg. 3 & $\begin{array}{l}\text { "Necessidade de se pensar metodologias } \\
\text { de priorização e avaliação de projetos de } \\
\text { P\&D". }\end{array}$ \\
\hline 27, pg. 3 & $\begin{array}{l}\text { "O setor elétrico brasileiro carece de } \\
\text { estudos que apontem os resultados } \\
\text { alcançados". }\end{array}$ \\
\hline 28, pg. 10 & $\begin{array}{l}\text { "Para que haja um efetivo retorno dos } \\
\text { investimentos realizados, é necessário um } \\
\text { efetivo acompanhamento dos projetos e } \\
\text { dos seus resultados". }\end{array}$ \\
\hline
\end{tabular}

Fonte: Elaborado pelo autor

A partir do exposto no Quadro 3, com as sugestões que os trabalhos aqui apresentados recomendam para pesquisas futuras, percebe-se que as principais questões que se apontam para serem tratadas neste cenário são: uma efetiva mensuração dos resultados do programa, uma avaliação dos projetos como um todo e verificação da eficiência dos mesmos e, consequentemente, do Programa.

\section{Conclusão}

Reconhecido o papel da Inovação como um fenômeno fundamental para o desenvolvimento econômico (Schumpeter, 1942) e, com o intuito de promover a movação esse processo dentro do setor elétrico (fazer frente aos desafios tecnológicos), a Lei 9.991/2000 surge com a finalidade de desenvolver a inovação no setor, estimulando as empresas de energia elétrica a investir parte de sua receita em Projetos de P\&D.
A partir deste argumento, tem-se que o objetivo deste artigo foi desenvolver uma busca na literatura, através de um trabalho bibliométrico, sobre o que tem sido discutido a respeito do Programa de P\&D da ANEEL.

Com um total de 31 trabalhos encontrados ao longo dos 15 anos, foi possível verificar alguns pontos que auxiliam às conclusões deste trabalho, tais como: evolução histórica, tipologia, objetivos, metodologia, impactos e inter-relações.

Quanto à Evolução Histórica, no que tange à publicação dos trabalhos, verificou-se que, ao longo do tempo houve uma tendência de crescimento, especialmente em 2013, contudo surpreendida por duas quedas em número de publicação, nos anos de 2012 e 2014. A causa para este declínio no número de trabalhos nestes anos não pôde ser aqui inferida por falta de dados que suportem tal explicação.

Em um período de quinze anos apenas três trabalhos foram publicados em periódicos científicos, o restante (28) foi, em sua maior parte, fruto de trabalhos de conclusão de mestrado e pesquisas apresentadas em Congressos. Este achado traduz a necessidade existente da pesquisa de inovação no setor elétrico ser levada mais efetivamente para os meios científicos.

Em relação aos objetivos identificados nos trabalhos, destacam-se 3: Avaliação dos Projetos, Gestão dos Projetos e Análise da Política. Em relação ao primeiro, o que se pretendia era verificar os resultados e os impactos que os projetos analisados exerciam na empresa estudada. Este ponto merece destaque ao enfatizar que as pesquisas trabalharam, em sua maioria, com empresas específicas, olhando suas particularidades. Não foram realizadas pesquisas que contemplassem os projetos do setor elétrico como um todo.

Outro resultado que esta bibliometria forneceu foi a capacidade de reconhecer a instituição que mais pesquisa sobre este tema e, além disso, reconhecer o autor que contribui efetivamente para a sua disseminação. Gilberto de Martino Jannuzzi, professor da Unicamp, apresenta-se como o autor do trabalho mais citado dentre os que foram pesquisados, publicado em um periódico científico internacional com fator de impacto de 2,57. Este resultado demonstra que a pesquisa no setor tem, de fato, bastante impacto e relevância, e que sua continuidade e aprofundamento são necessários para o desenvolvimento da área e principalmente do setor. A instituição a qual Jannuzzi faz parte destacouse como a instituição que mais colabora para o 
desenvolvimento de pesquisas neste campo. Além do número de trabalhos encontrados, a Unicamp também se mostrou como intermediadora de cooperações entre outras instituições a fim de que o tema seja disseminado e não particularizado dentro da universidade.

Todavia, a rede de interações entre as instituições que desenvolveram pesquisas caracterizou-se como dispersa, pois não possui relações intensas entre os atores. Observaram-se apenas dois blocos de interações que, por sua vez, também não apresentam força em suas relações (caracterizada pela grossura da linha que liga os atores).

Alguns aspectos qualitativos também foram observados neste trabalho. O fato dos Projetos de P\&D desenvolvidos pelas empresas nem sempre apresentarem aplicabilidade ou funcionalidade pode caracterizá-lo apenas como uma invenção, não chegando ao patamar de inovação (Schumpeter, 1942), o que faz não cumprir o objetivo da lei previamente estipulado.

Outro aspecto que a pesquisa revela é quanto ao comodismo dos gestores em atuarem em empresas que, a princípio, não necessitavam inovar e, assim, mudar o paradigma da organização. Este fator relaciona-se com a própria discussão do tema Inovação quanto aos seus principais aspectos (Pavitt, 1990).

Também foramidentificadas nos trabalhos pesquisados quais as sugestões recomendados pelos autores para pesquisas futuras, e notou-se que a avaliação e mensuração dos resultados dos projetos em geral e, consequentemente do programa, destaca-se como a principal recomendação para trabalhos futuros. De fato avaliar a eficiência de uma política mostra-se como fundamental para o bom funcionamento da mesma.

As contribuições oriundas desta pesquisa encontram suporte no fato de possibilitar aos pesquisadores interessados na política de P\&D da ANEEL, enxergar, por exemplo, a instituição e o autor chaves para tal campo, bem como enxergar os principais objetivos desenhados nas pesquisas realizadas até então na área. Além disso, também possibilita ver as lacunas que ainda existem no desenvolvimento de estudos no que tange a este tema, como por exemplo estdar o setor como um todo, ao invés de olhar as empresas de energia elétrica de forma particular, isso pode auxiliar a dar uma visão geral sobre o processo de inovação implantado pelas empresas.
Além disso, o trabalho também fornece contribuições teóricas para a área de inovação, à medida que confirma a teoria com dados empíricos encontrados em um setor que está desenvolvendo uma política voltada para o processo inovador. Ademais, estudar inovação em um setor onde tem características de monopólio e onde o produto fornecid é considerado uma commoditie, é demasiadamente interessante para os estudiosos do tema.

\section{Referências}

ABRADE. Disponível em $\leq$ http://www.abradee. com.br $\geq$ Acesso em 2 de outubro de 2015 .

Agência Nacional de Energia Elétrica (Brasil).

Manual do programa de pesquisa e desenvolvimento tecnológico do setor de energia elétrica. Agência Nacional de Energia Elétrica. Brasília : ANEEL, 2008.

\section{ALMEIDA, J. A. J. Investigação Apreciativa integrada às práticas de Gestão do Conhecimento em P\&D no Setor Elétrico Brasileiro: o caso da Companhia Hidro Elétrica do São Francisco. Tese de Doutorado (Administração). Universidade Federal de Pernambuco, 2013.}

\section{ALMEIDA, J. A. J. P\&D no setor elétrico} brasileiro: um estudo de caso na Companhia Hidro Elétrica do São

Francisco. 97 f. Dissertação (Mestrado em Economia), Universidade Federal de Pernambuco, Recife,2008.

AMARAL, G. S. G. A Pesquisa e

Desenvolvimento no Setor Elétrico: uma investigação da Política Tecnológica para o setor com base na Teoria Evolucionária da mudança técnica. Dissertação de Mestrado (Engenharia da Produção). USP, 2012.

ANDERSSON, U.; MATS, F.; HOLM, U. The strategic impact of external networks: subsidiary performance and competence development in the multinational corporation. Strategic Management Journal, No. 23, pp. 979-996. 2002

ANEEL. Disponível em $\leq$ http:/ /www.aneel.gov.br $\geq$ Acesso em 2 de outubro de 2015. 
ATALAY, M.; ANAFARTA, N.; SARVAN, F.

The relationship between innovation and firm performance: An empirical evidence from Turkish automotive supplier industry. Procedia - Social and Behavioral Sciences, v. 75, p. 226-235. 2013

The development of technological capabilities. Technology and International Competitiveness. Washington: The World Bank. 1995.

BACELLAR, A.M. Agilidade na Contratação de Projetos de Pesquisa do Programa de Pesquisa e Desenvolvimento da ANEEL. Dissertação. Universidade Federal do Rio Grande do Sul. 2014.

BELL, M.; PAVIT'T, K. Technological accumulation and industrial growth: contrast between developed and developing countries. Industrial and Corporate Change, v. 2, n. 2, p. 157-210. 1993.

BRASIL. Lei no 9.991, de 24 de julho de 2000. Lei de investimentos em pesquisa e Desenvolvimento pelas empresas de energia elétrica. Diário Oficial [da] República Federativa do Brasil, Brasília, DF, 24 jul. 2000. Disponível em: http:/ /www2.camara.leg.br/ legin/fed/lei/2000/lei-9991-24-julho-2000-359823normaatualizada-pl.html. Acesso em: 30 mai. 2013

CAIAFFA, C. M.; Logística na Apropriação de resultados de projetos de pesquisa, desenvolvimento e inovação: estudo de caso do portfólio de projetos do Programa Regulado de P\&D\&I da Light/ ANEEL. Dissertação de Mestrado (Engenharia Industrial). PUC RJ. 2013.

CALANTONE, R. J.; CAVUSGILA, S. T.; ZHAO, Y. Learning orientation, firm innovation capability, and firm performance. Industrial Marketing Management Vol. 31, 515- 524. 2002.

CAMPOS, M. Z. C. R.; FERREIRA, M. A. T.; SILVA, S. M.; ANDREASSI, T. Transferência do conhecimento tecnológico gerado em projetos de P\&D no setor elétrico brasileiro: o caso Eletronorte. Revista Economia e Gestão. Vol. 9, n.21, 2009.

CONNELL, J.; EDGAR, G. C.; OLEX, B.; SCHOLL, R.; SHULMAN, T.; TIETJEN, R.

Troubling Successes and Good Failures: Successful New Product Development Requires Five Critical Factors. Engineering Management Journal Vol. 13 No. 4. 2001.
DORNELLAS, C. R. R; BUENO, A. S.; STRUM, A.; KRUGLIANSKAS, I.; FIKS, M. Gestão de P\&D no Setor Elétrico Brasileiro: Estudo de Caso na Duke Energy. V CITENEL, Belém, 2009.

DOSI, G. Sources, procedures and microeconomic effects of innovation. Journal of Economic Literature, Vol. 26, No. 3, pp. 1120-1171. 1988.

------. Learning, capability accumulation and firms differences: Evidence from latecomer steel. Industrial and Corporate Change, Vol. 12, No. 3, pp. 607-643. 2003

FERNANDINO, J.A; OLIVEIRA, J.L. Arquiteturas Organizacionais para a Área de P\&D em Empresas do Setor Elétrico Brasileiro. RAC, v. 14, n. 6, art. 5, pp. 1073-1093, 2010.

FIGUEIREDO, P. N. Technological learning and competitive performance. Northampton, MA; Cheltenham, UK: Edward Elgar. 2001.

FREEMAN, C. Japan: a New National System of Innovation? In: Dosi, G.; Freeman, C.; Nelson, R. R.; Silverberg, G; Soete, L. Technical Change and Economic Theory. Londres: Printer, 1988.

FREITAS, F. L.; FERREIRA, M. P.; MATSUO, T.K.; FORCELLINI, F.A.; OROFINO, M. A. R. Processo De Desenvolvimento De Produto: Aplicação Em Um Projeto De P\&D Dentro Do Programa Aneel. XXIV Seminários Nacional de Parques Tecnológicos e Incubadoras de Empresas. Belém-PA. 2014.

GOMES, R. D. M., JANNUZZI, G. M. Um estudo de caso para análise dos projetos de $\mathrm{P} \& \mathrm{D}$ das concessionárias reguladas pela Aneel. International Energy Initiative, 2003.

GOMES, Rodolfo Dourado Maia. Pesquisa $\&$ Desenvolvimento de Interesse Público e as Reformas no Setor Elétrico Brasileiro. Campinas: Faculdade de Engenharia Mecânica. Dissertação. Universidade Estadual de Campinas, 2002.

GOUlarT, F. M. F. Gestão De Projetos De Pesquisa E Desenvolvimento No Setor Elétrico Brasileiro. Dissertação de Mestrado. Universidade FUMEC. Minas Gerais. 2014.

GUEDES, C. F. B. Políticas públicas de estímulo a P\&D: uma avaliação dos resultados do 
programa regulado pela Agência Nacional de Energia Elétrica - ANEEL. Dissertação. Universidade de Brasília. 2010.

HENDERSON, R.; CLARK, K.. Architectural Innovation: The Reconfiguration of Existing Product Technologies and the Failure of Established Firms. Administrative Science Quarterly. 35 (1), 81-112. 1990.

INSEAD, Cornell University, WIPO. The Global Innovation Index 2014: The Human Factor In innovation, Fontainebleau, Ithaca, and Geneva. 2014.

JANNUZZI, G. M. Public Goods and Restructuring of the Brazilian Power Sector: Energy Efficiency, R\&D and Low Income Programs ACEEE Summer Study on Energy Efficiency in Buildings: "Efficiency and Sustainability”. Asilomar, California, 20-25 August 2000 .

JANNUZZI, G. M. Power sector reforms in Brazil and its impacts on energy efficiency and research and development activities. Energy Policy, v. 33, pp. 1753-1762, 2005.

JUNIOR, E. I.; RIBEIRO, C. G.; FURTADO, A. T.; SILVA, G. Quão efetivas são políticas tecnológicas de cunho impositivas? Evidências do programa de P\&D capitaneadas por uma empresa do setor elétrico brasileiro. Revista Ciencias Estratégicas (Colombia), Vol. 21, No. 29, pp. 87-104, 2013.

LALL, S. Technological capabilities and industrialization. World Development, Vol. 20, pp. 165-186. 1992.

MAISONNAVE, P. R. A Contextualização da Inovação na Área de Pesquisa e Desenvolvimento das Empresas do Setor Elétrico Brasileiro. Dissertação de Mestrado (Administrção). PUC-RJ. 2008.

MAISONNAVE, P. R.; ROCHA-PINTO, S. R. Uma Análise Fenomenológica a respeito da percepção da Inovação nos Investimentos de Pesquisa e Desenvolvimento do Setor Elétrico Brasileiro. EnANPAD, Rio de Janeiro, 2008.

MASSAGUER, P. X. R.; SALLES-FILHO, S. L. M.; BIN, A.; ZEITOUM, C.; COSTA, J. P.; ARANTES, F.; CAMPOS, F. R. Avaliação de projetos de
Pesquisa e Desenvolvimento: influências e desafios do contexto do setor elétrico brasileiro. ALTEC, 2013.

NEVEZ, N. Critérios de Avaliação e Seleção de Projetos para o Programa de P\&D da Aneel. Dissertação de Mestrado (Tecnologia). Universidade Federal Tecnológica do Paraná, 2011.

\section{OECD - ORGANISATION FOR ECONOMIC} CO-OPERATION AND DEVELOPMENT.

Manual de Oslo: diretrizes para coleta e interpretação de dados sobre inovação. 2005. Disponível em: < http://www.oei.es/salactsi/oslo2.pdf>

PAVITT, K. What we know about the strategic management of technology. California

Management Review, v. 32, n. 3, p. 17-26, 1990.

PEREIRA, J. A.; CANCIGLIERI JUNIOR, O.; LIMA, J. P.; SILVA, S. B. QFD Application on Developing R\&D Project Proposal for the Brazilian Electricity Sector: A Case Study System Assets Monitoring and Control for Power Concessionaires. 2013.

PEREIRA, J. A.; CANCIGLIERI, O.

Multidisciplinary Systems Concepts Applied to R\&D

Projects Promoted by Brazilian Electricity Regulatory

Agency (ANEEL). 19th ISPE International

Conference on Concurrent Engineering, At

Trier, Germany. 2012.

POMPERMAYER, F. M. et al. Impactos

Econômicos e Tecnológicos do Programa

de P\&D Regulado pela ANEEL. In:

POMPERMAYER, F. M.;DE NEGRI, F.;

CAVALCANTE, L. R. (org). Inovação tecnológica no setor elétrico brasileiro: uma avaliação do programa de P\&D regulado pela ANEEL. Brasília: Ed. IPEA, 2011.

POMPERMAYER, F. M. et al. Impactos Qualitativos do Programa de P\&D Regulado pela ANEEL. In: POMPERMAYER, F. M.; DE NEGRI, F.; CAVALCANTE, L. R. (org). Inovação tecnológica no setor elétrico brasileiro: uma avaliação do programa de P\&D regulado pela ANEEL. Brasília: Ed. IPEA, 2011.

POMPERMAYER, F. M. et al. Rede de pesquisa formada pelo programa de $P \& D$ regulado pela ANEEL: abrangência e características. In: POMPERMAYER, F. M.;DE NEGRI, F;; 
CAVALCANTE, L. R. (org). Inovação tecnológica no setor elétrico brasileiro: uma avaliação do programa de $P \& D$ regulado pela ANEEL. Brasília: Ed. IPEA, 2011.

PRICE, D. P.; STOICA, M.; BONCELLA, R. J. The relationship between innovation, knowledge, and performance in family and non-family firms: an analysis of SMEs. Journal of Innovation and Entrepreneurship, 2, 14. 2013.

SANTOS, J.G.; SOUZA, C.G.; CASTRO, A.C. Outputs do Programa de P\&d da Aneel: um Estudo Bibliométrico. XI Simpósio de Excelência em Gestão e Tecnologia. 2014.

SCHUMPETER, J.A. Capitalism, Socialism and Democracy. New York: Harper. 1942

SILVA JUNIOR, R. G.; PROCOPIUK, M.; QUANDT, C. O. A Pesquisa e Desenvolvimento na Estratégia Competitiva das Concessionárias do Setor Elétrico Brasileiro. XII Simpósio de Administração da Produção, Logística e Operações Internacionais, FGV- EASP, 2009.

SPLITTER, K.; ROSA, C. A.; BORBA, J. A. Uma Análise das Características dos Trabalhos "Ditos" Bibliométricos Publicados no Enanpad entre 2000 e 2011. EnANPAD. Rio de Janeiro, 2012.

TÁVORA, L.; SICSÚ, A.; FURTADO, A. T.; RIBEIRO, C. G.; JUNIOR, E.I.; PEREIRA, V.G.; SILVA, G. Da Pesquisa e Desenvolvimento à inovação: um estudo de caso numa empresa do setor elétrico do nordeste brasileiro. ALTEC. 2013.

TÁVORA, Luciana E. da Mota. Inovação, ciência e tecnologia no Brasil: proposta de avaliação de projetos de $\mathbf{P} \& \mathrm{D}$ no setor elétrico. Tese. Universidade Federal de Pernambuco. Recife-PE, 2010 .

TEIXEIRA, M. L. M. ; IWAMOTO, H. M.; MEDEIROS, A. L. Estudos Bibliométricos (?) Em Administração: Discutindo A Transposição De Finalidade. Administração: Ensino E Pesquisa Rio De Janeiro V. 14 No 3 P. 423-452, 2013

VISACRO, S. Programa de P\&D da ANEEL: Concepções, Falácias e Novos Desafios. Seminários Nacional de Transmissão de Energia Elétrica. Curitiba, 2005.
VONORTAS, N.; ZIRULIA, L. Strategic technology alliances and network. Economics of Innovation and New Technology, Vol. 24, No. 5, 490-509, 2015.

ZIVIANI, F.; FERREIRA, M. A. T. Práticas de gestão da inovação no setor elétrico brasileiro: a percepção dos gerentes de projetos de P\&D. ALETC, 2013. 